\title{
Protective Effects of Intralipid and Caffeic Acid Phenyl Esther (CAPE) on Neurotoxicity Induced by Ethanol in Rats
}

\author{
Seyit Kagan BASARSLAN ${ }^{1}$, Arif OSUN², Serkan SENOL ${ }^{3}$, Murat KORKMAZ², Umit OZKAN¹, Ibrahim KAPLAN ${ }^{4}$ \\ ${ }^{1}$ Mustafa Kemal University, Medical Faculty, Department of Neurosurgery, Hatay, Turkey \\ 2Dumlupınar University, Medical Faculty, Department of Neurosurgery, Kutahya, Turkey \\ ${ }^{3}$ Medeniyet University, Goztepe Education and Training Hospital, Department of Pathology, Istanbul, Turkey \\ ${ }^{4}$ Dicle University, Medical Faculty, Department of Biochemistry, Diyarbakır, Turkey
}

\section{ABSTRACT}

AIM: Ethanol causes oxidative degradation of the mitochondrial genome in the brain. This effect could contribute to the development of brain injury in some alcoholic patients. We investigated the protective effect of caffeic acid phenyl esther (CAPE) and intralipid (IL) on oxidative stress and neurotoxicity induced by ethanol intake.

MATERIAL and METHODS: The forty-eight rats were randomly divided into seven groups. Ethanol was administered for acute toxicity. IL and CAPE were administered immediately after ethanol intake. Total oxidant status (TOS), total antioxidant status (TAS), and oxidative status index (OSi) were evaluated and histologic examination of cerebellum and brain tissue with Hematoxylin-Eosin and immuno-histochemical dyes was performed.

RESULTS: In the ethanol group, TAS levels were significantly lower than the other groups and this finding indicates that the toxic effect of ethanol reduces antioxidant levels. In the ethanol group, TOS levels were significantly higher than the other groups. These results showed that ethanol induced oxidative stress. IL treatment increased TAS levels, and CAPE decreased TOS levels against ethanol toxicity. There was correlation between TAS and TOS levels. Also, histopathologic results confirmed these biochemical results.

CONCLUSION: CAPE and IL treatment could be effective course of therapy to enhance therapeutic efficacy and may provide a promising approach for the treatment of neurotoxicity and oxidative stress induced by ethanol in clinic.

KEYWORDS: Ethanol, Caffeic acid phenyl ester, Intralipid, Neurotoxicity

\section{INTRODUCTION}

$\mathrm{E}$ thanol, is a colorless liquid with the structural formula $\mathrm{CH}_{3} \mathrm{CH}_{2} \mathrm{OH}$, often abbreviated as $\mathrm{C}_{2} \mathrm{H}_{5} \mathrm{OH}$ or $\mathrm{C}_{2} \mathrm{H}_{6} \mathrm{O}$. It is also used as a psychoactive drug and is one of the oldest recreational drugs still used by humans. Ethanol can cause alcohol intoxication when consumed.

Alcoholic patients can develop not only liver lesions but also pancreatitis, brain damage, peripheral neuropathy, cardiomyopathy, and skeletal muscle myopathy $(18,23)$. Reactive oxygen species (ROS) and free radicals are generated during ethanol metabolism, causing oxidative stress and lipid peroxidation in the liver, brain, heart and skeletal muscles $(1,6,18,21)$.

Mitochondria are major targets for ethanol toxicity in the liver, brain, heart, skeletal muscles, and exocrine pancreas (4). Ramachandran et al (23) showed in their study that utilized cultured fetal rat cortical neurons that ethanol elicits a rapid onset of oxidative stress, which culminates in mitochondrial mediated apoptotic cell death. Animal studies have found that long-term ethanol intoxication is not necessary to cause brain 
damage. Studies have shown that as little as a few days of intoxication can lead to neuronal loss in several brain areas of adult rats (8).

Metabolic and physiological processes produce reactive oxygen species and either enzymatic or nonenzymatic antioxidative mechanisms may cause harmful oxidative reactions in organisms. Shift of oxidative/antioxidative balance towards the oxidative status is due to the increase in oxidants and decrease in antioxidants (10).

Antioxidant molecules can prevent and/or inhibit these harmful reactions (11). Oxidative stress index (OSi) is accepted as the indicator of the degree of oxidative stress and was calculated as the ratio between TOS and TAS (TOS/TAS).

In recent years, several reports have suggested that caffeic acid phenyl esther (CAPE), active substance of propolis, is a natural composite with anti-inflammatory, antioxidant, immuno-modulatory, antimycosis and anticarcinogenic effects (2). The antioxidant activity of propolis extract is mainly attributed to its flavonoid content, which is capable of scavenging free radicals and thereby protection against lipid peroxidation. Propolis also induces the activation of antioxidant enzymes such as superoxide dismutase and catalase against free radicals (3).

Intralipid (IL) contains mainly triglycerides with saturated and unsaturated fatty acids. The unsaturated lipids could act as ROS scavengers. IL could also act directly on cell membranes, and induce structure alterations leading to a decrease of the ROS release in the extracellular medium (16).

In this study, we investigated the protective effect of CAPE and $\mathrm{IL}$ on oxidative stress and neurotoxicity induced by ethanol intake.

\section{MATERIAL and METHODS}

\section{Animals, Care and Nutrition}

A total of forty-eight female Wistar Albino rats weighing 200$250 \mathrm{~g}$ were kept under laboratory conditions with a 12-hour light/dark cycle and a room temperature of $21 \pm 3^{\circ} \mathrm{C}$. Standard pellet food was used for feeding and all animals had free access to water. The study was approved by the Necmettin Erbakan University Experimental Medical Research Center's Experimental Animals Ethics Committee.

\section{Animals and Treatment}

The forty-eight rats were randomly divided into seven groups $(n=6)$ as control $(C)$, Ethanol $(E)$, IL, CAPE, IL plus ethanol $(\mathrm{IL}+\mathrm{E})$, CAPE plus ethanol (CAPE+E) and IL plus CAPE plus ethanol $(\mathrm{IL}+\mathrm{CAPE}+\mathrm{E})$ groups. The rats were administered ethanol ( $3 \mathrm{mg} / \mathrm{kg}$ oral), IL (18.6 mL/ $/ \mathrm{kg}$ oral) and with CAPE $(10 \mu \mathrm{mol} / \mathrm{kg}$ intraperitoneal). IL and CAPE were administered immediately after $\mathrm{E}$ administration. Rats were sacrificed under ketamine/xylazine $(60 / 5 \mathrm{mg} / \mathrm{kg})$ anesthesia. Brain tissue of the rats was removed and divided longitudinally into two sections. One section was stored under $-70^{\circ} \mathrm{C}$ for biochemical analysis and the other part was kept in formaldehyde solution for histologic examination.

\section{Biochemical Analysis}

\section{The total antioxidant status (TAS)}

TAS of supernatant fractions was measured by automated and colorimetric method developed by Erel O. $(10,11)$. This method allows producing the most potent biological radicals such as hydroxyl radicals. In the experiment, the ferrous ion solution was mixed with hydrogen peroxide and potent radicals such as dianisidinyl radical cations produced by the hydroxyl radicals were developed. By this way, the antioxidative effect of the material was measured against the potent-free radical reactions started by the produced hydroxyl radicals. The experiment has excellent sensitivity values lower than $3 \%$. The total antioxidant response results were mentioned as nmol Trolox equivalent/mg protein.

\section{The total oxidant status (TOS)}

The TOS of supernatant fractions was measured by automated and colorimetric method developed by Erel O. $(10,11)$. Ferrous ion-o-dianisidine complex is oxidized to ferric ion by the oxidants present in the sample. Glycerol molecules, which were profusely present in the reaction medium, increase the oxidation reaction. In the acidic medium, ferric ion produces a colored complex with xylenol orange and spectrophotometric methods can measure the color intensity that is related to the total amount of oxidant molecules present in the sample. The measurement was calibrated with hydrogen peroxide and the results were presented as nmol $\mathrm{H}_{2} \mathrm{O}_{2}$ equivalent/ng protein.

\section{Histopathologic Analysis}

\section{Immunohistochemical procedures}

Immunohistochemical examination was performed on a Leica Bond-Max automated IHC/ISH platform (Leica Microsystems Inc, Buffalo Grove, Illinois). Four-micrometer paraffin sections were dewaxed in a Bond Dewax solution, rehydrated in alcohol and Bond Wash solution (Leica Microsystems). Antigen retrieval was performed using a high-pH (ER2) retrieval solution for 15 minutes followed by endogenous peroxidase blocking for 5 minutes on the machine. Antimouse monoclonal antibody Bcl-2 (C-2: sc-7382, Santa Cruz Biotechnology, Inc. in dilution 1:200), anti-mouse monoclonal antibody Bax (B-9: sc-7480, Santa Cruz Biotechnology, Inc. in dilution 1:100) and anti-mouse caspase-3 (CPP32) monoclonal antibody (clone JHM62, Leica Biosystems Ltd, Newcastle) at 1:50 dilution were applied for 60 minutes at room temperature. Detection was performed using the Bond Polymer Refine Red Detection system (Leica Microsystems) with a 15-minute postprimary step followed by 25 minutes of incubation with alkaline phosphatase-linked polymers. Sections were then counterstained with hematoxylin on the machine, dehydrated in alcohols, and mounted with mounting medium (Sakura Finetek USA Inc, Torrance, California).

Histopathologists who were unaware of the experimental study groups evaluated the specimens. Apoptotic cells were counted in a blind fashion microscope fields under 400X magnification. The average number of stained neurons for each set of ten fields were calculated and mentioned as the number of the positive cells/high-power field. 


\section{Histological examination}

The brain and cerebellum specimens were dehydrated in alcohol and embedded in paraffin after immersed individually in $10 \%$ neutral buffered formaldehyde. $4 \mu \mathrm{m}$ sections were deparaffinized and stained with hematoxylin and eosin. The specimens were examined in random order under blindfold conditions with light microscopy. Inflammation, oedema, congestion, degeneration, necrosis and necrobiosis were evaluated. After the results were graded, all of the groups were compared with each other.

\section{Statistical Analysis}

The data for the biochemical parameters were analyzed by ANOVA, followed by the post hoc Tukey test and Dunnet T3. All data was presented using SPSS Windows 20.0 (IBM SPSS Statistics Data editor). A value of $p<0.05$ was considered statistically significant.

\section{RESULTS}

\section{Biochemical Results}

TAS levels: In ethanol group, TAS levels were lower than other groups and this finding indicates that toxic effect of ethanol reduces antioxidant level. Comparing the E+CAPE, E+IL and $E+C A P E+I L$ groups with the $E$ group revealed that TAS levels were lower in the E and E+CAPE groups than the other groups. This finding showed that IL treatment is effective on the oxidative stress due to ethanol toxicity (Figure 1).

TOS levels: In the $E$ and $E+I$ groups, TOS levels were significantly higher than other groups. The most significant result was the decrease of TOS level in the CAPE group. This result showed that CAPE decreases the oxidative stress level (Figure 2). The oxidative stress index (OSi) revealed that ethanol causes significant oxidative stress (Figure 3).

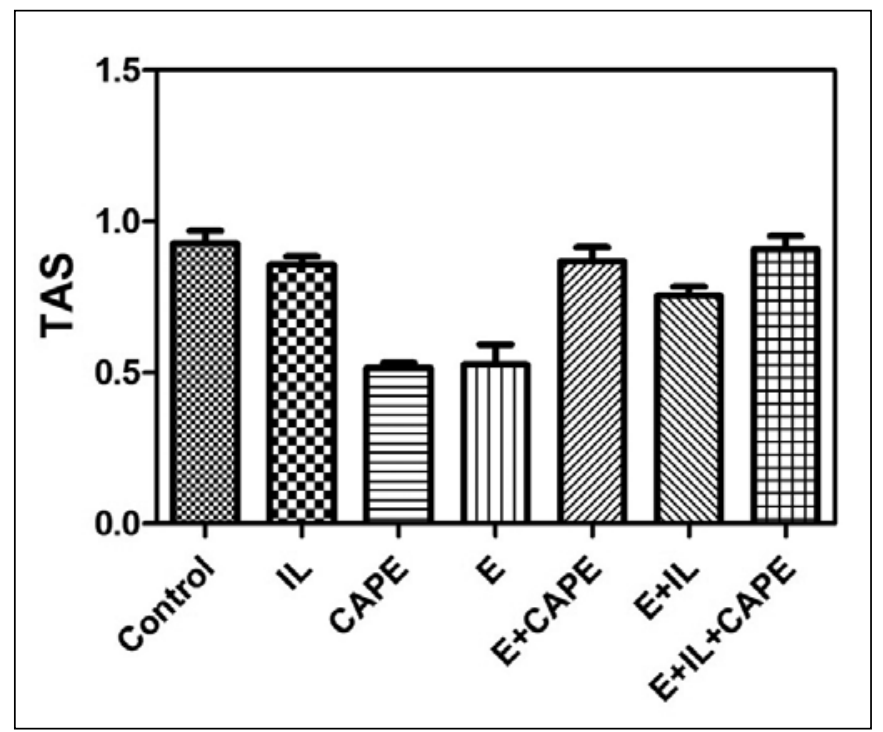

Figure 1: Evaluating the TAS levels showed that there is significant difference between ethanol group with control, CAPE, IL, E+CAPE, $\mathrm{E}+\mathrm{IL}$ and $\mathrm{E}+\mathrm{CAPE}+\mathrm{IL}$ groups.
The comparison of the post-medication TAS, TOS, and OSi levels in brain tissues is summarized in Table I.

\section{Histological Findings}

\section{Hematoxylin and eosin (H\&E):}

The brain and cerebellum specimens were examined under the light microscope (X200 and X400) for inflammation, oedema, congestion, degeneration, necrosis and necrobiosis findings.

\section{Scoring:}

Semi-quantative grading was performed as:

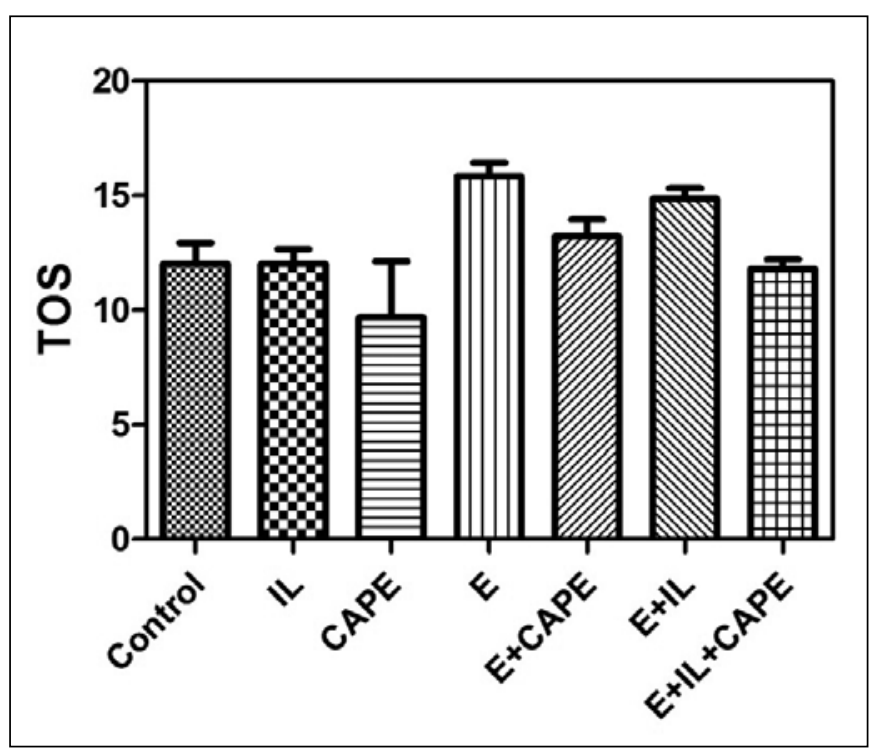

Figure 2: Evaluating the TOS levels showed that there is significant difference between ethanol group with control, CAPE, $\mathrm{IL}, \mathrm{E}+\mathrm{CAPE}, \mathrm{E}+\mathrm{IL}$ and $\mathrm{E}+\mathrm{CAPE}+\mathrm{IL}$ groups.

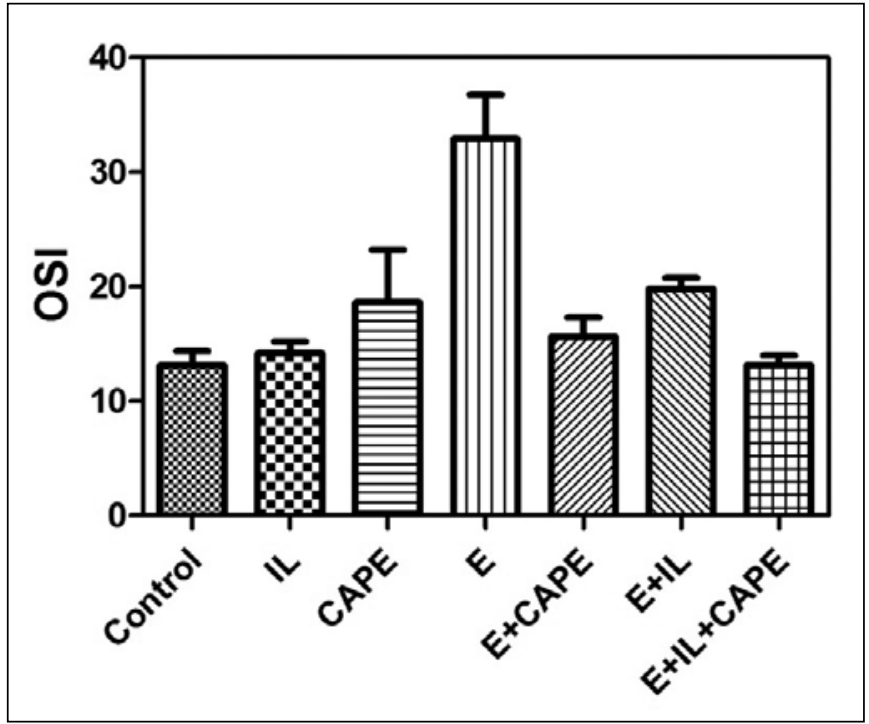

Figure 3: Oxidative stress index (OSi) revealed that ethanol causes significant oxidative stress. 
Table I: Comparison of Post-Medication TAS, TOS, and OSi Levels in Brain Tissues

\begin{tabular}{lccc}
\hline & TAS & TOS & OSi \\
\hline Control & $0.92 \pm 0.10$ & $12.05 \pm 2.20$ & $13.15 \pm 3.00$ \\
\hline CAPE & $0.51 \pm 0.03$ & $9.69 \pm 5.46$ & $18.66 \pm 10.21$ \\
\hline IL & $0.85 \pm 0.06$ & $12.05 \pm 1.64$ & $14.20 \pm 2.65$ \\
\hline Ethyl Alcohol & $0.52 \pm 0.18^{*}$ & $15.84 \pm 1.73^{*}$ & $32.92 \pm 10.93^{*}$ \\
\hline Ethyl Alcohol+CAPE & $0.86 \pm 0.11$ & $13.22 \pm 1.82$ & $15.62 \pm 4.19$ \\
\hline Ethyl Alcohol+IL & $0.75 \pm 0.07$ & $14.85 \pm 1.14$ & $19.82 \pm 2.32$ \\
\hline Ethyl Alcohol +IL+CAPE & $0.90 \pm 0.10^{+}$ & $11.81 \pm 0.99^{+}$ & $13.17 \pm 1.99^{+}$ \\
\hline
\end{tabular}

Data are presented as mean $\pm S D$.

The mean difference is significant at the level of $0.05(p<0.05)$.

*Compared with the Ethyl Alcohol group, the TAS values in the Control, IL, E+CAPE, and E+IL+CAPE groups were significantly higher.

${ }^{*}$ Compared with the Ethyl Alcohol group, the TOS values in Control, IL, CAPE, and E+IL+CAPE groups were significantly lower.

${ }^{*}$ Compared with the Ethyl Alcohol group, the OSI values in Control, IL, E+CAPE and E+IL+CAPE groups were significantly lower.

+ Compared with the Ethyl Alcohol+IL+CAPE group, the TAS values in the Ethyl Alcohol and CAPE groups were significantly lower.

+ Compared with the Ethyl Alcohol+IL+CAPE group, the TOS values in the Ethyl Alcohol and E+IL groups were significantly higher.

+ Compared with the Ethyl Alcohol+IL+CAPE group, the OSI values in the Ethyl Alcohol group was significantly higher.

Ethyl Alcohol (E), IL (Intralipid), CAPE (Caffeic acid phenethyl ester).

Table II: Score Results of Degeneration Findings

\begin{tabular}{lc}
\hline Groups & Total Score / 69 \\
\hline 1. Control group & $6 / 69$ \\
\hline 2. ETHANOL & $37 / 69$ \\
\hline 3. CAPE & $9 / 69$ \\
\hline 4. IL & $11 / 69$ \\
\hline 5. E+CAPE & $17 / 69$ \\
\hline 6. E+IL & $32 / 69$ \\
\hline 7. E+CAPE+IL & $21 / 69$ \\
\hline
\end{tabular}

Oedema and congestion: None: 0 , Minimal: 1, Moderate: 2, Severe: 3

Inflammation: Negative: 0, Positive: 1

Degeneration: None: 0 , Minimal: 1, Moderate: 2 Severe: 3

Necrosis and necrobiosis: None: 0, Minimal: 1, Moderate: 2, Severe: 3

The score results were summarized in Table II.

Neurons were normal in the control group (Figure 4A, B). In the ethanol group, there was significant neuronal degeneration with the findings of a dark pyknotic nucleus, vacuolation and shrunken cytoplasm (Figure 5A), inflammation (Figure $5 \mathrm{~B}$ ), significant oedema, congestion, degeneration (Figure 5C) and necrosis (Figure 5D). E+IL group evaluation showed that neuronal degeneration was less than the ethanol group but there was no statistical significance ( $p>0.05$ ). In E+CAPE (Figure 6) and E+IL+CAPE groups, all of the degeneration findings were significantly less than the ethanol group $(p<0.05)$.

\section{Immunohistochemical findings:}

Seven rats were evaluated in each group. Caspase-3, bcl-2 and bax antibodies were used for apoptotic cell counting. Ten randomized microscope fields (X200) were counted (Figure $7 A)$. There was a significant increase of apoptotic cells in ethanol group (Figure 7B). Examination of $\mathrm{E}+\mathrm{IL}, \mathrm{E}+\mathrm{CAPE}$ and $E+I L+C A P E$ groups showed a significant decrease in apoptotic cells (Figure 7C,D) when comparing with the ethanol alone group $(\mathrm{p}<0.05)$.

\section{DISCUSSION}

There are several mechanisms that have been proposed to explain E-related brain damage. It is well known that ethanol, when administered acutely in a pharmacologically relevant dose, selectively and potently inhibits the function of N-methyl-D-aspartate (NMDA) receptors. Increased calcium influx through NMDA receptors is tightly coupled to uptake into mitochondria and causes the production of reactive oxygen species that interfere with the function of mitochondria and plasma membranes $(9,20)$. It is well known that ethanol metabolism causes oxidative stress. Mansouri et al. (19) showed that acute ethanol intake oxidatively damages and reduces mitochondrial DNA in mouse liver, brain, heart and skeletal muscles. So, it is clear that ethanol intake causes oxidative stress in the liver and extra-hepatic tissues.

The present study showed TAS levels were significantly lower than other groups and this finding indicates that toxic effect of $E$ reduces antioxidant levels in $E$ group. Also, in the $E$ group, TOS levels were significantly higher than the other groups. These results showed that $E$ significantly induced oxidative stress.

Death from $E$ consumption is possible when blood alcohol level reaches $0.4 \%$. A blood level of $0.5 \%$ or more is commonly 


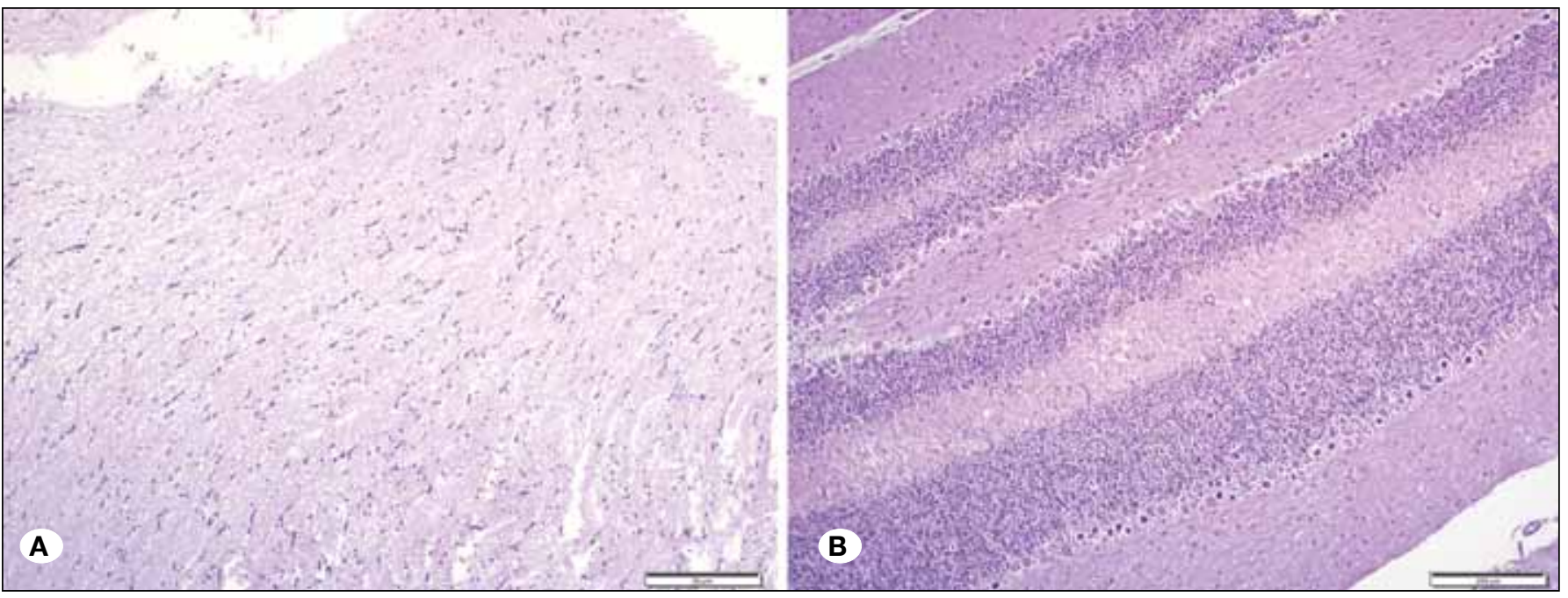

Figure 4: Normal brain (A) and cerebellum (B) tissue of rat (HE, X100).
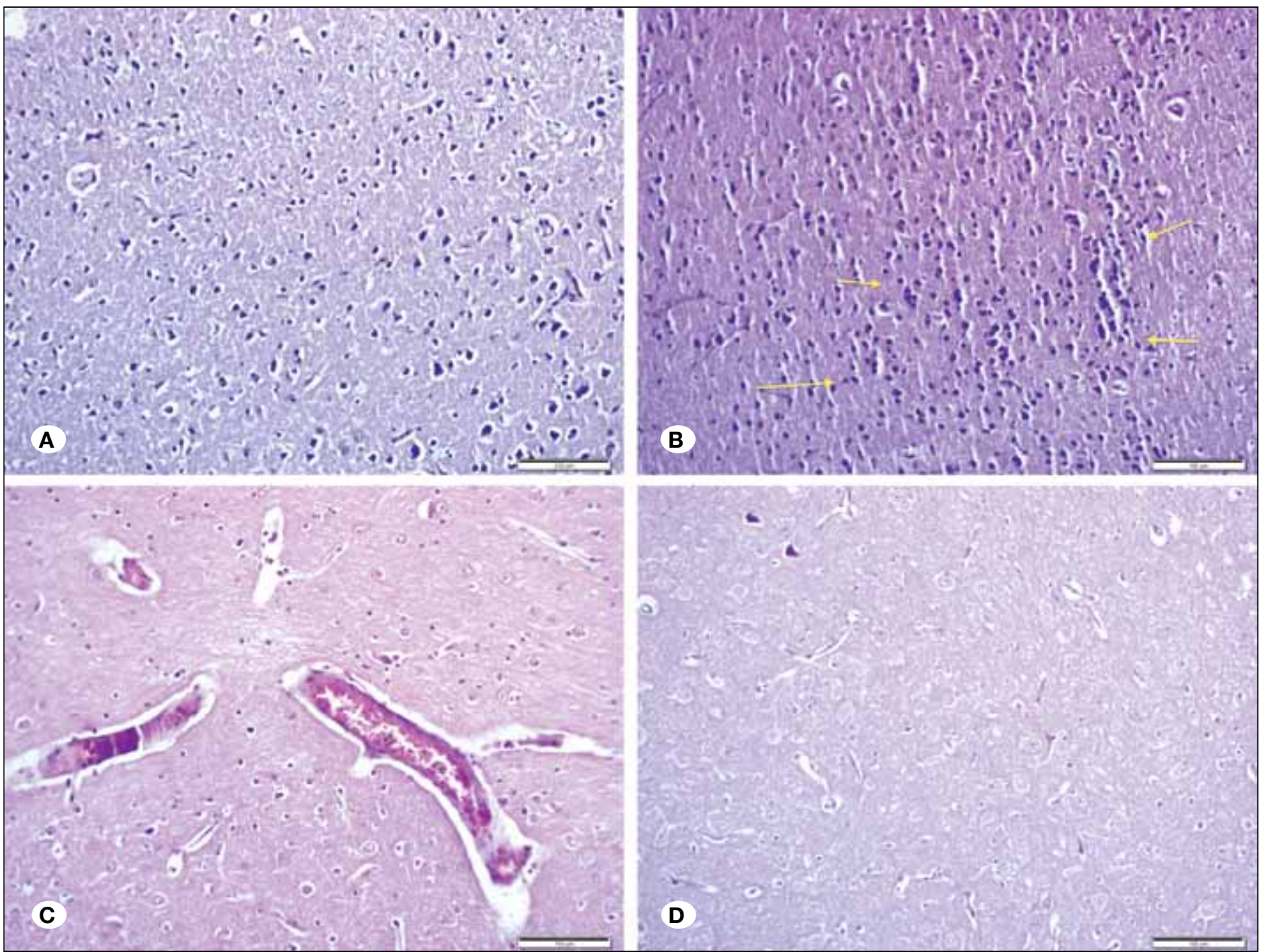

Figure 5: Histologic examination of Ethanol group; A) there was significant neuronal degeneration with the findings of dark pyknotic nucleus, vacuolation and shrunken cytoplasm, B) significant inflammation, C) significant oedema, congestion and degeneration and D) necrosis (HE, X200). 


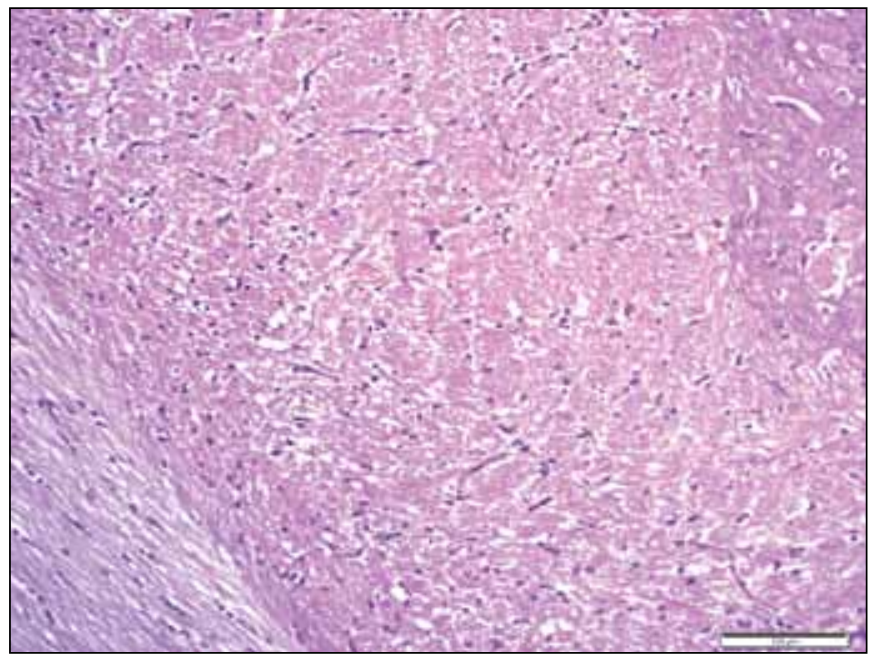

Figure 6: $\mathrm{E}+\mathrm{IL}+\mathrm{CAPE}$ group; all of the degeneration findings were significantly fewer than ethanol group (HE, X200). fatal. Levels of even less than $0.1 \%$ can cause intoxication, with unconsciousness often occurring at $0.3-0.4 \%$ (29). Neurotrophic factors, particularly neurotrophins, play a vital role in neuronal survival and maturation, and are important in regulating naturally occurring cell death through apoptotic mechanisms. Reduced levels of these trophic factors or deprivation of trophic-like effects from glial cells can induce alterations in the pattern of neuronal synaptic connections or cause cell death. Recent studies suggest that chronic exposure to ethanol can reduce the availability of brainderived neurotrophic factor and alter its receptor function (7).

Using transcranial magnetic stimulation, Ravaglia et al. (24) showed that chronic alcoholics had a significant prolongation of central motor conduction time compared with controls, but that there was no correlation between intensity and duration of abuse. Volumetric magnetic resonance imaging (MRI) analysis revealed thalamic and mammillary body atrophy with relative sparing of medial temporal lobe structures. However,

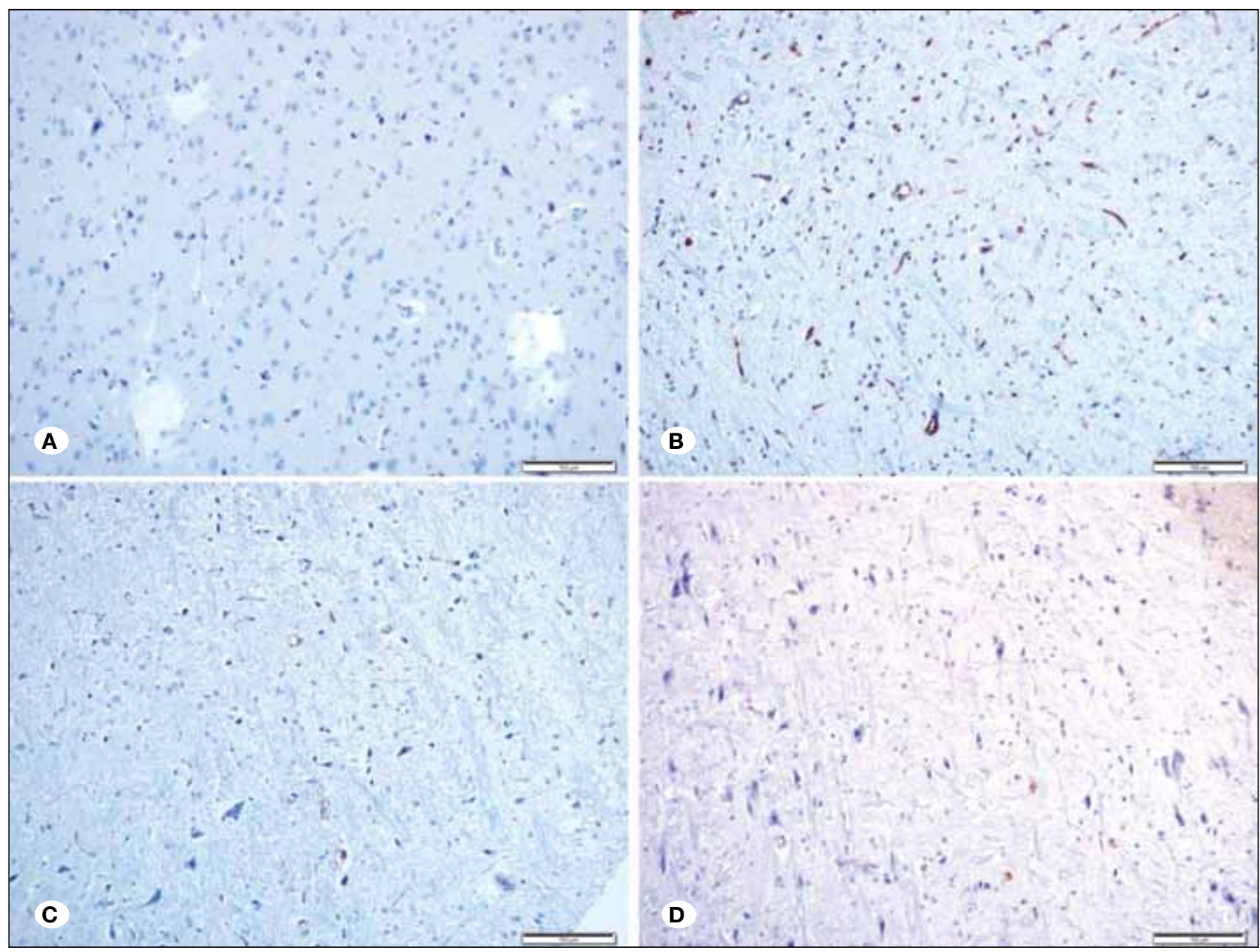

Figure 7: Immunohistochemical evaluation; A) Normal brain tissue of the rat, B) Ethanol group revealed that there is significant apoptosis, C,D) E+CAPE+IL group showed mild apoptosis (IHC X200). 
fluorodeoxyglucose-positron emission tomography data from the same patients showed relative hypometabolism in the diencephalic grey matter and medial temporal regions (25). Alcohol-related neuronal loss has been documented in specific regions of the cerebral cortex (superior frontal association cortex), hypothalamus and cerebellum (15).

Intralipid is a brand name for the first safe fat emulsion for human use. It is used as one of the major component in parenteral nutrition for patients who are unable to have sufficient nutrition via an oral diet. Recently, it has been used to resuscitate lethal local anesthetic drug toxicity (12). Moreover, several animal studies supported that the use of IL as an antidote could treat bupivacaine toxicity (26). The exact mechanisms of antidotal action are not clear yet. Only the "lipid sink" mechanism provides a potential explanation (28). Intralipids probably move fat-soluble drugs away from the site of toxicity, and thereby, may alleviate toxic effects of the fat-soluble drugs. Basarslan et al. (5) reported that IL is a promising safe therapy on neurotoxicity caused by malathion which is an organophosphate pesticide. There is a very limited number of studies on using IL on intoxications in the literature. Also, there is no study on the protective effects of IL on ethanol-induced neurotoxicity. In this study, it has been shown that IL protects the brain tissue from the oxidative stress damage of ethanol. This finding is very important terms both beneficial effects of IL in E intoxication and the effects of alcohol intake with fatty foods.

3,4-dihydroxycinnamic acid (CAPE) is a non-flavanoid catecholic compound present in many plants and is present in the diet as part of fruits, tea and wine. It is known that CAPE has a broad spectrum of pharmacological activities including anti-inflammatory, antioxidant and immunomodulatory effects (14). Pharmacology studies have also shown that CAPE exerts a protective effect against hydrogen peroxide-induced oxidative damage in the brain (22). Although many of these effects have been partially related to 5-lipoxigenase inhibition, the mechanisms involved in the protective activities of CAPE have not yet been fully understood.

Uzar et al. (27) reported that CAPE significantly reduced the activities of antioxidant enzymes and the level of malondialdehyde in the cerebellum of rats subjected to methotrexate. Ginis et al. (13) reported that pretreatment with CAPE might protect brain tissue against ifosfamide-induced central neurotoxicity. Huang et al. (17) reported that CAPE may provide a promising approach for the treatment of acrolein-related neurodegenerative diseases with decreasing oxidative stress such as Alzheimer's disease.

This study revealed that CAPE has a protective effect against ethanol-induced neurotoxicity and combination with IL treatment amplifies the protection. Also, there is no study on the protective effect of CAPE on ethanol-induced neurotoxicity in literature. Therefore, this study suggests that CAPE may provide a promising approach for the treatment of alcohol toxicity.

\section{- CONCLUSION}

CAPE and IL treatment could be effective course of therapy to enhance therapeutic efficacy and may provide a promising approach for the treatment of neurotoxicity and oxidative stress induced by ethanol in clinic.

\section{REFERENCES}

1. Adachi J, Asano M, Ueno $Y$, Reilly M, Mantle D, Peters TJ,

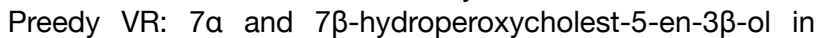
muscle as indices of oxidative stress: Response to ethanol dosage in rats. Alcohol Clin Exp Res 24: 675-681,2007

2. Alp $\mathrm{H}$, Aytekin I, Esen $\mathrm{H}$, Alp A, Buyukbas S, Basarali $\mathrm{K}$, Hatipoglu NK, Kul S: Protective effects of caffeic acid phenethyl ester, ellagic acid, sulforaphan and curcuma on malathion induced damage in lungs, liver and kidneys in an acute toxicity rat model. Revue Méd Vét 162: 333-340,2011

3. Attia AA, El-Mazoudy $\mathrm{RH}$, El-Shenawy NS: Antioxidant role of propolis extract against oxidative damage of testicular tissue induced by insecticide chlorpyrifos in rats. Pesticide Biochemistry and Physiology 103: 87-93,2012

4. Baker RC, Kramer RE: Cytotoxicity of short-chain alcohols. Annu Rev Pharmacol Toxicol 39:127-150,1999

5. Basarslan SK, Alp H, Senol S, Evliyaoglu O, Ozkan U: Is intralipid fat emulsion a promising therapeutic strategy on neurotoxicity induced by malathion in rats? Eur Rev Med Pharmacol Sci 18(4):471-476, 2014

6. Calabrese V, Renis M, Calderone A, Russo A, Reale S, Barcellona ML, Rizza V: Stress proteins and SH-groups in oxidant-induced cellular injury after chronic ethanol administration in rat. Free Radic Biol Med 24: 1159-1167,1998

7. Climent E, Pascual M, Renau-Piqueras J, Guerri C: Ethanol exposure enhances cell death in the developing cerebral cortex: Role of brain-derived neurotrophic factor and its signaling pathways. J Neurosci Res 68:213-225,2002

8. Collins MA, Corso TD, Neafsey EJ: Neuronal degeneration in rat cerebrocortical olfactory regions during subchronic "binge" intoxication with ethanol: Possible explanation for olfactory deficits in alcoholics. Alcohol Clin Exp Res 20: 284-292,1996

9. Dodd PR, Beckmann AM, Davidson MS, Wilce PA: Glutamatemediated transmission, alcohol, and alcoholism. Neurochem Int 37:509-533,2000

10. Erel O: A new automated colorimetric method for measuring total oxidant status. Clinical Biochemistry 38: 1103-1111,2005

11. Erel O: A novel automated method to measure total antioxidant response against potent free radical reactions. Clinical Biochemistry 37: 112-119,2004

12. Foxall G, McCahon R, Lamb J, Hardman JG, Bedforth NM: Levobupivacaine-induced seizures and cardiovascular collapse treated with Intralipid. Anaesthesia 62: 516-518,2007

13. Ginis Z, Ozturk G, Albayrak A, Kurt SN, Albayrak M, Fadillioglu E: Protective effects of caffeic acid phenethyl ester on ifosfamide-induced central neurotoxicity in rats. Toxicol Ind Health. Toxicol Ind Health 32(2):337-343, 2016

14. Gulcin I: Antioxidant activity of caffeic acid. Toxicology 217:213-220, 2006

15. Harper CG, Kril JJ, Daly J: Are we drinking our neurones away? Br Med J 294:534-536,1987

16. Hartert MM, Dupont GD, Hans P, Deby C, Lamy M: Protective activity of propofol, Diprivan ${ }^{\circledR}$ and intralipid against active oxygen species. Mediators of Inflammation 7: 327-333,1998 
17. Huang Y, Jin M, Pi R, Zhang J, Chen M, Ouyang Y, Liu A, Chao $X$, Liu P, Liu J, Ramassamy C, Qin J: Protective effects of caffeic acid and caffeic acid phenethyl ester against acroleininduced neurotoxicity in HT22 mouse hippocampal cells. Neurosci Lett 22(535):146-151,2013

18. Kurose I, Higuchi H, Kato S, Miura S, Ishii S: Ethanol-induced oxidative stress in the liver. Alcohol Clin Exp Res 20:7785,1996

19. Mansouri A, Demeilliers C, Amsellem S, Pessayre D, Fromenty $\mathrm{B}$ : Acute ethanol administration oxidatively damages and depletes mitochondrial DNA in mouse liver, brain, heart, and skeletal muscles: Protective Effects of Antioxidants. JPET 298:737-743,2001

20. Matsumoto I, Burke L, Inoue Y, Wilce PA: Two models of ethanol withdrawal kindling. Nihon Arukoru Yakubutsu Igakkai Zasshi 36:53-64,2001

21. Nordmann R, Ribie're C, Rouach $\mathrm{H}$ : Implication of free radical mechanisms in ethanol-induced cellular injury. Free Radic Biol Med 12: 219-240,1992

22. Pereira P, Oliveira P, Ardenghi P, Rotta L, Henriques J, Piccada $\mathrm{J}$ : Neuropharmacological analysis of caffeic acid in rats. Basic Clin Pharmacol Toxicol 99:374-378,2006

23. Ramachandran V, Watts LT, Maffi SK, Chen J, Schenker S, Henderson G: Ethanol-induced oxidative stress precedes mitochondrially mediated apoptotic death of cultured fetal cortical neurons. JNR 74: 577-588,2003
24. Ravaglia S, Costa A, Ratti MT, Savoldi F, Bo P, Moglia A: Cognitive impairment and central motor conduction time in chronic alcoholics. Funct Neurol 17:83-86,2002

25. Reed LJ, Lasserson D, Marsden P, Stanhope N, Stevens T, Bello F, Kingsley D, Colchester A, Kopelman MD: FDGPET findings in the Wernicke-Korsakoff syndrome. Cortex 39:1027-1045,2003

26. Rosenblatt MA, Abel M, Fischer GW, Itzikovich CJ, Eisenkraft JB: Successful use of a $20 \%$ lipid emulsion to resuscitate a patient after a presumed bupivacaine-related cardiac arrest. Anesthesiology 105: 217-218,2006

27. Uzar E, Koyuncuoglu HR, Uz E, Yilmaz HR, Kutluhan S, Kilbas S, Gultekin F: The activities of antioxidant enzymes and the level of malondialdehyde in cerebellum of rats subjected to methotrexate: Protective effect of caffeic acid phenethyl ester. Mol Cell Biochem 291:63-68,2006

28. Weinberg GL, Vadeboncouer T, Ramaraju GA, Garcia-Amaro MF, Cwik MJ: Pretreatment or resuscitation with a lipid infusion shifts the dose-response to bupivacaine-induced asystole in rats. Anesthesiology 88: 1071-1075,1998

29. Yost DA: Acute care for alcohol intoxication. Be prepared to consider clinical dilemmas. Postgrad Med 112(6):14-16, 21 22, 25-26, 2002 